\title{
Inventário de macroliquens epífitos sobre árvores utilizadas na arborização urbana em Curitiba, Paraná, Brasil: Subsídio para biomonitoramento urbano
}

\author{
Sionara Eliasaro* \\ Patrícia Wolf Veiga \\ Cristine Gobel Donha \\ Lucas Nogueira \\ Laboratório de Liquenologia, Departamento de Botânica, Setor de Ciências Biológicas \\ Universidade Federal do Paraná, CEP 81531-970, Curitiba - PR, Brazil \\ *Autor para correspondência \\ sionara@ufpr.br
}

Submetido em 06/02/2009

Aceito para publicação 06/08/2009

\section{Resumo}

Foi analisada a composição de comunidades de macroliquens epífitos sobre as seguintes espécies arbóreas utilizadas na arborização urbana em Curitiba: Acer negundo, Lagerstroemia indica, Ligustrum lucidum, Parapiptadenia rigida, Cassia leptophylla, Syagrus romanzoffian, Tabebuia alba, Tabebuia chrysotricha, Tabebuia heptaphylla e Tipuana tipu. Registrou-se um total de 84 espécies, sendo 14 citadas pela primeira vez para o estado do Paraná e Flavoparmelia soredians citada pela primeira vez para o Brasil. Parmeliaceae foi a família melhor representada com 45 espécies distribuídas em nove gêneros; seguida de Physciaceae com 24 espécies distribuídas em seis gêneros. As espécies arbóreas nativas apresentaram maior riqueza de espécies de liquens e maior número de espécies exclusivas do que as espécies arbóreas exóticas. A maior riqueza de espécies foi encontrada em Tabebuia chrysotricha, com 62 espécies, seguida de Syagrus romanzoffiana com 47 espécies. Candelaria concolor, Canoparmelia crozalsiana, Canoparmelia texana, Dirinaria applanata, Dirinaria confluens, Heterodermia obscurata, Myelochroa lindmanii, Parmotrema pilosum, Physcia poncinsii, Punctelia borreri, Punctelia reddenda, Pyxine subcinerea, Ramalina celastri e Ramalina peruviana são indicadas como espécies de macroliquens com maior potencial em futuros estudos de biomonitoramento da qualidade do ar na cidade de Curitiba.

Unitermos: bioindicadores, Parmeliaceae, Physciaceae

\section{Abstract}

Inventory of epiphytic macrolichens on trees used in urban arborization in Curitiba, Paraná, Brazil. The floristic composition of epiphytic macrolichens on the following tree species used in urban arborization in Curitiba was analysed: Acer negundo, Lagerstroemia indica, Ligustrum lucidum, Parapiptadenia rigida, Cassia leptophylla, Syagrus romanzoffiana, Tabebuia alba, Tabebuia chrysotricha, Tabebuia heptaphylla, and Tipuana tipu. A total of 84 species are reported, from which 14 are recorded for the first time in Paraná State 
and Flavoparmelia soredians is recorded for the first time in Brazil. Parmeliaceae was the best represented family, with 45 species distributed in nine genera, followed by Physciaceae with 24 species in six genera. The native tree species showed greater lichen species richness and a higher number of exclusive lichen species than the exotic tree species. The highest lichen species richness was found in Tabebuia chrysotricha with 62 taxa, followed by Syagrus romanzoffiana with 47. Candelaria concolor, Canoparmelia crozalsiana, Canoparmelia texana, Dirinaria applanata, Dirinaria confluens, Heterodermia obscurata, Myelochroa lindmanii, Parmotrema pilosum, Physcia poncinsii, Punctelia borreri, Punctelia reddenda, Pyxine subcinerea, Ramalina celastri and Ramalina peruviana are suggested as macrolichen species with the greatest potential for future biomonitoring studies of air quality in Curitiba.

Key words: bioindicators, Parmeliaceae, Physciaceae

\section{Introdução}

A poluição atmosférica constitui-se em um dos principais problemas dos grandes centros urbanos. A utilização de bioindicadores permite o monitoramento da poluição atmosférica a curto, médio e longo prazo sem a necessidade de utilizar equipamentos sofisticados e de alto custo, além de mostrar os resultados da ação de poluentes sobre organismos vivos, uma abordagem relevante para a determinação do impacto da tecnologia sobre a biosfera (Seaward, 2002).

Numerosos estudos utilizando os liquens como biondicadores têm sido realizados desde que Nylander em 1866 correlacionou a poluição do ar com o desaparecimento de espécies de liquens (Sommerfeldt e John, 2000). Grande parte destes trabalhos está relacionada ao mapeamento da comunidade liquênica epifítica em uma dada área/cidade (e.g. Giordani, 2007; Jeran et al., 2007; Munzi et al., 2007; Martins et al., 2008; Poličnik et al., 2008). Estes mapeamentos podem ser feitos utilizando informações sobre a comunidade liquênica total, procurando detectar reduções na distribuição natural das espécies, sendo um tipo de estudo indicado para monitoramento em áreas pequenas e em torno de uma fonte poluidora (Pignata, 1998).

Uma variação deste método, que permite estudos em áreas maiores, consiste na utilização de um número limitado de espécies que sejam boas indicadoras de qualidade do ar (Pignata, 1998). A validade deste segundo método é dependente dos critérios utilizados na seleção das espécies. Para se determinar quais espécies de liquens são indicadas é fundamental o conhecimento prévio da composição da comunidade liquênica na área a ser investigada, quais espécies de liquens podem ocorrer sobre diferentes espécies de forófitos, e quais espécies de forófitos podem apresentar uma comunidade liquênica diversificada (Pignata, 1998). Além disto, para que o trabalho possa ter uma aplicação prática, devem ser selecionadas espécies que possam ser reconhecidas por não especialistas (Geebelen e Hoffmann, 2001).

Em geral, em áreas industriais ou centrais de núcleos urbanos, os únicos substratos disponíveis para o desenvolvimento de liquens são as árvores utilizadas em arborização urbana. As comunidades liquênicas que se desenvolvem sobre estes forófitos, mesmo quando em locais com poucos poluentes atmosféricos, possuem uma composição diferente das que ocorrem em árvores de parques ou bosques adjacentes, apresentando espécies que se adaptam a este ambiente.

A cidade de Curitiba apresenta uma significativa diversidade na composição de espécies arbóreas utilizadas na arborização de ruas. Milano et al. (1992) registram 103 espécies, no entanto 77,6\% das árvores plantadas pertencem a somente 10 espécies. De acordo com Milano et al. (1992), dentre estas 10 espécies, seis são nativas e correspondem a $31,5 \%$ de todas as árvores plantadas nas ruas de Curitiba: Cassia leptophylla Vog. (4,9\%), vulgarmente conhecida por Canafístula, Tabebuia alba (Cham.) Sadwith (7,3\%) e Tabebuia chrysotricha (Mart. Ex DC) Stand. (6,8\%), espécies conhecidas por Ipê-amarelo, Tabebuia heptaphylla (Vell.) Toledo (7,0\%), o Ipê-roxo, Parapiptadenia rigida (Benth.) Brenan (4,4\%), o Angico, e Syagrus romanzoffiana (Cham.) Glassm. (1,1\%), conhecido por Jerivá. Ainda de acordo com Milano et al. (1992), as outras quatro espécies são exóticas e correspondem a quase metade de todas as árvores plantadas nas ruas 
de Curitiba (46,1\%): Acer negundo Linn. (5,2\%), conhecido por Ácer, Lagerstroemia indica Linn. (19\%), a Extremosa, Ligustrum lucidum Ait. (15,4\%), popularmente Alfeneiro, e Tipuana tipu (Benth.) Kuntze (6,5\%), a Tipuana.

O conhecimento de quais espécies de liquens podem se desenvolver sobre estas árvores, se existe preferência de liquens por forófito, bem como a avaliação de quais destas espécies arbóreas podem apresentar uma comunidade liquênica diversificada, são pré-requisitos para a elaboração de projetos de biomonitoramento utilizando liquens em Curitiba. Neste trabalho objetivamos fornecer subsídios a futuros estudos de biomonitoramento da qualidade do ar na cidade de Curitiba utilizando macroliquens epífitas.

\section{Materiais e Métodos}

Os liquens foram coletados, de acordo com a metodologia usual em liquenologia (Brodo et al., 2001), sobre o tronco das seguintes espécies arbóreas: Acer negundo, Cassia leptophylla, Lagerstroemia indica, Ligustrum lucidum, Parapiptadenia rigida, Syagrus romanzoffiana, Tabebuia alba, Tabebuia chrysotricha, Tabebuia heptaphylla e Tipuana tipu. Foram amostrados 20 indivíduos de cada espécie arbórea com exceção de $L$. indica que por perder o córtex periodicamente, demandou maior esforço amostral, sendo analisados 27 indivíduos. Todas as árvores amostradas localizavam-se em ruas asfaltadas, com pouco tráfego, nos seguintes bairros residenciais: Abranches, Bacacheri, Barreirinha, Batel, Bom Retiro, Boqueirão, Jardim das Américas, Jardim Social, Santo Inácio e Seminário. Os exemplares foram incorporados ao herbário UPCB da Universidade Federal do Paraná.

Cada exemplar foi analisado sob microscópio estereoscópico (20-50X), realizando-se observações morfológicas detalhadas. Nos exemplares que apresentaram estruturas reprodutivas do micobionte, foram feitos cortes à mão livre destas estruturas, os quais foram analisados sob microscópio óptico (400-1000X). Para a caracterização e identificação de metabólitos secundários de importância taxonômica foram realizados: testes de coloração de córtex e medula de acordo com
Elix (1994); observação do talo sob lâmpada UV e, quando necessário, cromatografia de camada delgada, segundo técnica padronizada por Culberson e Ammann (1979) e Elix e Ernst-Russell (1993).

A riqueza florítica foi considerada como o número total de espécies de liquens ocorrentes nos indivíduos analisados (Martins et al., 2008). Para estimar a similaridade da composição de espécies liquênicas entre as diferentes espécies arbóreas foi utilizado o Índice de similaridade de Sorensen (Cs), baseado na presença comum de espécies.

\section{Resultados}

Foram encontradas 84 espécies de macroliquens distribuídas em 24 gêneros e nove famílias (Tabela 1). Parmeliaceae foi a família melhor representada com 45 espécies distribuídas em nove gêneros; seguida de Physciaceae com 24 espécies distribuídas em seis gêneros. Ramalinaceae, Teloschistaceae e Usneaceae apresentaram, cada uma, três espécies em um único gênero; Candelariaceae e Collemataceae apresentaram, cada uma, duas espécies em um único gênero; enquanto Lobariaceae e Coccocarpiaceae apresentaram apenas uma espécie.

Candelaria concolor (Dick.) B. Stein, C. fibrosa (Fr.) Müll. Arg., Coccocarpia palmicola (Spreng.) L. Arv. \& D. Gall., Parmotrema subisidiosum (Müll. Arg.) Hale, Heterodermia comosa (Eschw) Follm. \& Redón, H. flabellata (Fée) Awasthi, H. galactophylla (Tuck.) Trev., Hyperphyscia adglutinata (Flörke) May. \& Poelt, H. pandani (H. Magn.) Moberg, H. syncolla (Nyl.) K. Kalb, H. tuckermanii (Lynge) Moberg, Physcia erupens Moberg, P. poncinsii Hue e Pyxine subcinerea Stirton são registradas pela primeira vez para o estado do Paraná. Flavoparmelia soredians está sendo citada pela primeira vez para o Brasil.

Tabebuia chrysotricha foi a espécie arbórea que apresentou maior riqueza de espécies sobre seu córtex (62), sendo seis destas encontradas exclusivamente nesta árvore: Hypotrachyna pluriformis (Nyl.) Hale, Parmotrema catarinae Hale, Parmotrema melanothrix (Mont.) Hale, Heterodermia comosa, Heterodermia corallophora (Tayl.) Skorepa e Usnea sp. 3. 
O forófito com a segunda maior riqueza de espécies foi Syagrus romanzoffiana (47), apresentando duas espécies exclusivas: Parmelinopsis damaziana (Zahlbrl.) Hale e Parmotrema mellissii (C. W. Dodge) Hale. Os forófitos Ligustrum lucidum e Tipuana tipu apresentaram o mesmo valor de riqueza florística com 43 espécies, porém apenas Ligustrum lucidum apresentou espécies exclusivas: Heterodermia galactophylla e Phaeophyscia cf. adiastola (Essl.) Essl.. Já Tabebuia alba (37) apresentou duas espécies não encontradas em outro forófito da área de estudo: Parmotrema recipiendum (Nyl.) Hale e Usnea sp. 2.

Acer negundo e Tabebuia heptaphylla apresentaram o mesmo valor de riqueza florística (35 taxa), entretanto apenas Tabebuia heptaphylla possuía uma espécie exclusiva, Coccocarpia palmicola. Parapiptadenia rigida (34) apresentou apenas uma espécie exclusiva, Bulbotrix isidiza (Nyl.) Hale. Cassia leptophylla (25) e Lagerstroemia indica (21) apresentaram o menor número de espécies de liquens, sendo que $C$. leptphylla não possuiu espécies exclusivas e Teloschistes chrysophthalmus (L.) Th. Fr. foi coletado exclusivamente em ramos finos de $L$. indica.

Candelaria concolor, Canoparmelia crozalsiana (Bouly de Les.) Elix \& Hale, Parmotrema pilosum (Stiz.) Krog \& Swinscow, Canoparmelia texana (Tuck) Elix \& Hale, Dirinaria applanata (Fée) Awasthi, Heterodermia obscurata (Nyl.) Trev., Myelochroa lindmanii (Lynge) Elix \& Hale, Physcia poncinsii, Punctelia borreri, Punctelia reddenda e Ramalina peruviana Ach. apresentaram-se indiferentes em relação à forófito, ocorrendo sobre todas as espécies amostradas.

Dirinaria confluens (Fr.) Awasthi, Pyxine subcinerea e Ramalina celastri (Spreng.) Krog \& Swinscow também se revelaram pouco exigentes em relação à forófito, não sendo encontradas, respectivamente, sobre Tipuana tipu, Tabebuia heptaphylla e Cassia leptophylla.

TABELA 1: Macroliquens sobre córtex de espécies arbóreas utilizadas na arborização urbana em Curitiba (1 - Lagerstroemia indica, 2 - Ligustrum lucidum, 3 - Tabebuia alba, 4 - Tabebuia heptaphylla, 5 Tabebuia chrysotricha, 6-Tipuana tipu, 7-Acer negundo, 8-Cassia leptophylla,9-Parapiptadenia rigida, 10 - Syagrus romanzoffiana).

\begin{tabular}{|c|c|c|c|c|c|c|c|c|c|c|}
\hline Macrolíquen & 1 & 2 & 3 & 4 & 5 & 6 & 7 & 8 & 9 & 10 \\
\hline \multicolumn{11}{|l|}{ Candelariaceae } \\
\hline Candelaria concolor (Dick.) B. Stein & $\mathrm{X}$ & $\mathrm{X}$ & $\mathrm{X}$ & $\mathrm{X}$ & $\mathrm{X}$ & $\mathrm{X}$ & $\mathrm{X}$ & $\mathrm{X}$ & $\mathrm{X}$ & $\mathrm{X}$ \\
\hline C. fibrosa (Fr.) Müll. Arg. & & $\mathrm{X}$ & & & & & & & $\mathrm{X}$ & $\mathrm{X}$ \\
\hline \multicolumn{11}{|l|}{ Collemataceae } \\
\hline Leptogium cyanescens (Rabenh.) Körber & & $\mathrm{X}$ & & & & $\mathrm{X}$ & $\mathrm{X}$ & & $\mathrm{X}$ & \\
\hline Leptogium sp & & & & & & $\mathrm{X}$ & $\mathrm{X}$ & & & \\
\hline \multicolumn{11}{|l|}{ Coccocarpiaceae } \\
\hline Coccocarpia palmicola (Spreng.) L. Arv. \& D. Gall. & & & & $\mathrm{X}$ & & & & & & \\
\hline \multicolumn{11}{|l|}{ Lobariaceae } \\
\hline Pseudocyphelaria aurata (Ach.) Vain. & & $\mathrm{X}$ & $\mathrm{X}$ & $\mathrm{X}$ & $\mathrm{X}$ & $\mathrm{X}$ & $\mathrm{X}$ & $\mathrm{X}$ & & \\
\hline \multicolumn{11}{|l|}{ Parmeliaceae } \\
\hline Bulbotrix isidiza (Nyl.) Hale & & & & & & & & & $\mathrm{X}$ & \\
\hline B. ventricosa (Hale \& Kurok.) Hale & & & & & $\mathrm{X}$ & & & & $\mathrm{X}$ & $\mathrm{X}$ \\
\hline Canoparmelia caroliniana (Nyl.) Elix \& Hale & & & $\mathrm{X}$ & & $\mathrm{X}$ & & & & & \\
\hline C. crozalsiana (Bouly de Les.) Elix \& Hale & $\mathrm{X}$ & $\mathrm{X}$ & $\mathrm{X}$ & $\mathrm{X}$ & $\mathrm{X}$ & $\mathrm{X}$ & $\mathrm{X}$ & $\mathrm{X}$ & $\mathrm{X}$ & $\mathrm{X}$ \\
\hline C. texana (Tuck) Elix \& Hale & $\mathrm{X}$ & $\mathrm{X}$ & $\mathrm{X}$ & $\mathrm{X}$ & $\mathrm{X}$ & $\mathrm{X}$ & $\mathrm{X}$ & $\mathrm{X}$ & $\mathrm{X}$ & $\mathrm{X}$ \\
\hline Flavoparmelia soredians (Nyl.) Hale & & $\mathrm{X}$ & $\mathrm{X}$ & $\mathrm{X}$ & $\mathrm{X}$ & $\mathrm{X}$ & & & $\mathrm{X}$ & $\mathrm{X}$ \\
\hline Hypotrachyna livida (Tayl.) Hale & & & & & $X$ & & & & & $\mathrm{X}$ \\
\hline H. osseoalba (Vain.) Y.S. Park \& Hale & & & & & $\mathrm{X}$ & & & & & $\mathrm{X}$ \\
\hline H. pluriformis (Nyl.) Hale & & & & & $\mathrm{X}$ & & & & & \\
\hline Myelochroa lindmanii (Lynge) Elix \& Hale & $\mathrm{X}$ & $\mathrm{X}$ & $\mathrm{X}$ & $\mathrm{X}$ & $\mathrm{X}$ & $\mathrm{X}$ & $\mathrm{X}$ & $\mathrm{X}$ & $\mathrm{X}$ & $\mathrm{X}$ \\
\hline
\end{tabular}


Parmelinella wallichiana (Tayl.) Elix \& Hale

Parmelinopsis damaziana (Zahlbrl.) Hale

P. minarum (Vain.) Elix \& Hale

Parmotrema austrosinense (Zahlbr.) Hale

P. catarinae Hale

P. cetratum (Ach.) Hale

P. chinense (Osbeck) Hale \& Ahti

P. commensuratum (Hale) Hale

P. conferendum Hale

P. consors (Nyl.) Krog \& Swinscow

$P$. eciliatum (Nyl.) Hale

P. homotomum (Nyl.) Hale

P. internexum (Nyl.) Hale

P. macrocarpum (Pers.) Hale

P. melanohtrix (Mont.) Hale

P. mellissii (C. W. Dodge) Hale

P. muelleri (Vain.) O. Blanco, A. Crespo, Divakar, Elix \& Lumbsch

P. permutatum (Stirt.) Hale

P. pilosum (Stiz.) Krog \& Swinscow

P. recipiendum (Nyl.) Hale

P. reticulatum (Tayl.) M. Choisy

P. sancti-angeli (Lynge) Fleig

$P$. simulans (Hale) Hale

P. subcaperatum (Kremp.) Hale

P. subisidiosum (Müll. Arg.) Hale

P. subsumptum (Nyl.) Hale

P. tinctorum (Nyl.) Hale

Punctelia borreri (Sm.) Krog

$P$. constantimontium Sérus

P. microsticta (Müll. Arg.) Krog

P. negata (Nyl.) Krog

$P$. reddenda (Stirt.) Krog

$P$. riograndensis (Lynge) Krog

P. rudecta (Ach.)Hale \& Fletcher

P. subrudecta (Nyl.) Krog

$\begin{array}{cccccccccc} & \mathrm{X} & \mathrm{X} & & \mathrm{X} & \mathrm{X} & & & \mathrm{X} & \mathrm{X} \\ & & & & & & & & & \mathrm{X} \\ \mathrm{X} & \mathrm{X} & & \mathrm{X} & \mathrm{X} & & \mathrm{X} & & \mathrm{X} & \mathrm{X} \\ & & & & \mathrm{X} & & & & & \\ \mathrm{X} & & \mathrm{X} & \mathrm{X} & \mathrm{X} & & & \mathrm{X} & \mathrm{X} & \mathrm{X} \\ \mathrm{X} & \mathrm{X} & \mathrm{X} & & \mathrm{X} & \mathrm{X} & \mathrm{X} & \mathrm{X} & \mathrm{X} & \\ \mathrm{X} & \mathrm{X} & \mathrm{X} & \mathrm{X} & \mathrm{X} & \mathrm{X} & \mathrm{X} & & & \\ & \mathrm{X} & & & \mathrm{X} & \mathrm{X} & & & & \mathrm{X} \\ & \mathrm{X} & & & & & \mathrm{X} & & & \mathrm{X} \\ & & \mathrm{X} & & \mathrm{X} & & \mathrm{X} & & \mathrm{X} & \\ & & & & \mathrm{X} & \mathrm{X} & & & & \\ & \mathrm{X} & & & \mathrm{X} & \mathrm{X} & \mathrm{X} & & & \\ & & & \mathrm{X} & \mathrm{X} & & & & \mathrm{X} \\ & & & \mathrm{X} & & & & & \end{array}$

$x$
$x$

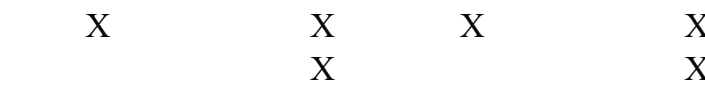

$\begin{array}{llllllllll}X & X & X & X & X & X & X & X & X & X\end{array}$

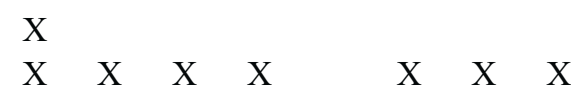

$\begin{array}{llllllll}X & X & X & X & & X & X & X \\ & & X & & & & & X\end{array}$

$\mathrm{X} \quad \mathrm{X}$

$\mathrm{X}$

$\mathrm{X}$

X $x$ $\mathrm{X}$

Physciaceae

Dirinaria applanata (Fée) Awasthi

D. confluens (Fr.) Awasthi

D. picta (Sw.) Clem \& Shear

Heterodermia albicans (Pers.) Swins \& Krog

H. casarettiana (Mass.) Trev.

H. comosa (Eschw) Follm. \& Redón

H. corallophora (Tayl.) Skorepa

H. flabellata (Fée) Awasthi

H. galactophylla (Tuck.) Trev.

H. leucomelos (L.) Poelt

H. lutescens (Kurok.) Follm.

H. obscurata (Nyl.) Trev.

H. vulgaris (Vain.) Follm.

Hyperphyscia adglutinata (Flörke) May. \& Poelt

H. pandani (H.Magn.) Moberg

$\begin{array}{cccccccccc}\mathrm{X} & \mathrm{X} & \mathrm{X} & \mathrm{X} & \mathrm{X} & \mathrm{X} & \mathrm{X} & \mathrm{X} & \mathrm{X} & \mathrm{X} \\ \mathrm{X} & \mathrm{X} & \mathrm{X} & \mathrm{X} & \mathrm{X} & & \mathrm{X} & \mathrm{X} & \mathrm{X} & \mathrm{X} \\ & \mathrm{X} & \mathrm{X} & & \mathrm{X} & \mathrm{X} & \mathrm{X} & & \mathrm{X} & \mathrm{X} \\ & \mathrm{X} & & & & & \mathrm{X} & & & \\ & \mathrm{X} & & \mathrm{X} & \mathrm{X} & \mathrm{X} & \mathrm{X} & \mathrm{X} & & \mathrm{X} \\ & & & & \mathrm{X} & & & & & \\ & & & & \mathrm{X} & & & & & \\ \mathrm{X} & \mathrm{X} & \mathrm{X} & \mathrm{X} & \mathrm{X} & \mathrm{X} & \mathrm{X} & & \mathrm{X} & \\ & \mathrm{X} & & & & & & & & \\ & \mathrm{X} & \mathrm{X} & \mathrm{X} & \mathrm{X} & & & & & \\ & & & \mathrm{X} & & & & & & \\ \mathrm{X} & \mathrm{X} & \mathrm{X} & \mathrm{X} & \mathrm{X} & \mathrm{X} & \mathrm{X} & \mathrm{X} & \mathrm{X} & \mathrm{X} \\ & & \mathrm{X} & & & \mathrm{X} & & & & \\ & \mathrm{X} & & & \mathrm{X} & \mathrm{X} & \mathrm{X} & \mathrm{X} & & \mathrm{X} \\ & \mathrm{X} & & & \mathrm{X} & \mathrm{X} & \mathrm{X} & & & \end{array}$


H. syncolla (Nyl.) K. Kalb

H. tuckermanii (Lynge) Moberg

Phaeophyscia cf. adiastola (Essl.) Essl.

Physcia alba (Fée) Müll. Arg.

Ph. erupens Moberg

Ph. poncinsii Hue

Ph. sorediosa (Vain.) Lynge

Physcia sp.

Pyxine subcinerea Stirton

\section{Ramalinaceae}

Ramalina celastri (Spreng.) Krog \& Swinscow

Ramalina peruviana Ach.

Ramalina pusiola Müll. Arg.

\section{Usneaceae}

Usnea sp. 1

Usnea sp. 2

Usnea sp. 3

\section{Teloschistaceae}

Teloschistes crysophthalmus (L.) Th. Fr.

T. exilis (Sw.) Norm.

T. flavicans (Mich.) Vainio

Os maiores valores de similaridade florística na composição de espécies liquênicas, foram encontrados, respectivamente entre as seguintes espécies arbóreas exóticas: Ligustrum lucidum-Acer negundo $(0,82)$ e

$$
\begin{array}{cccccccccc} 
& \mathrm{X} & & & & \mathrm{X} & & & & \\
& \mathrm{X} & \mathrm{X} & \mathrm{X} & \mathrm{X} & \mathrm{X} & \mathrm{X} & \mathrm{X} & & \mathrm{X} \\
\mathrm{X} & & & & & & & & \\
& \mathrm{X} & \mathrm{X} & & \mathrm{X} & \mathrm{X} & & \mathrm{X} & \mathrm{X} & \mathrm{X} \\
& \mathrm{X} & \mathrm{X} & \mathrm{X} & \mathrm{X} & \mathrm{X} & \mathrm{X} & \mathrm{X} & & \\
\mathrm{X} & \mathrm{X} & \mathrm{X} & \mathrm{X} & \mathrm{X} & \mathrm{X} & \mathrm{X} & \mathrm{X} & \mathrm{X} & \mathrm{X} \\
& \mathrm{X} & & \mathrm{X} & & \mathrm{X} & & & \mathrm{X} & \\
& & & & & \mathrm{X} & & & & \mathrm{X} \\
\mathrm{X} & \mathrm{X} & \mathrm{X} & & \mathrm{X} & \mathrm{X} & \mathrm{X} & \mathrm{X} & \mathrm{X} & \mathrm{X}
\end{array}
$$

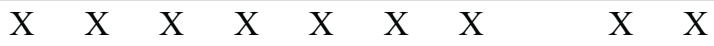
$X$ X $X$

$\mathrm{X}$

\begin{tabular}{|c|c|c|c|c|c|c|c|c|c|}
\hline Forófitos & 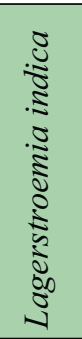 & 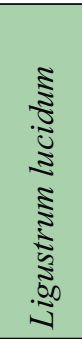 & $\begin{array}{c}\sqrt{3} \\
8 \\
8 \\
8 \\
8 \\
8 \\
8 \\
8 \\
8\end{array}$ & 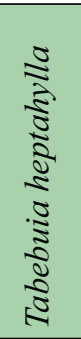 & 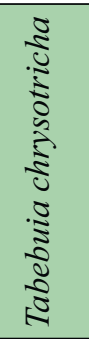 & 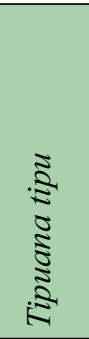 & 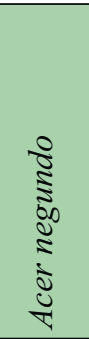 & 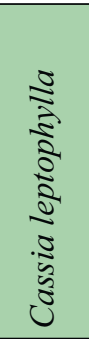 & 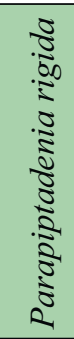 \\
\hline Ligustrum lucidum & 0,61 & & & & & & & & \\
\hline Tabebuia alba & 0,64 & 0,65 & & & & & & & \\
\hline Tabebuia heptaphylla & 0,65 & 0,63 & 0,66 & & & & & & \\
\hline Tabebuia chrysotricha & 0,50 & 0,65 & 0,69 & 0,59 & & & & & \\
\hline Tipuana tipu & 0,55 & 0,79 & 0,67 & 0,63 & 0,69 & & & & \\
\hline Acer negundo & 0,67 & 0,82 & 0,64 & 0,65 & 0,60 & 0,72 & & & \\
\hline Cassia leptophylla & 0,64 & 0,59 & 0,68 & 0,62 & 0,57 & 0,59 & 0,63 & & \\
\hline Parapiptadenia rigida & 0,64 & 0,57 & 0,70 & 0,57 & 0,63 & 0,60 & 0,58 & 0,61 & \\
\hline Syagrus romanzoffiana & 0,52 & 0,64 & 0,59 & 0,63 & 0,73 & 0,60 & 0,59 & 0,58 & 0,64 \\
\hline
\end{tabular}
$\mathrm{X}$

$\mathrm{X}$

\section{$\mathrm{X}$}

TABELA 2: Similaridade florística da composição de espécies liquênicas entre as espécies arbóreas utilizadas na arborização urbana de Curitiba. Índice de Sorensen. 


\section{Discussão}

A grande maioria $(94,1 \%)$ das espécies de macroliquens ocorrentes sobre as árvores utilizadas em arborização urbana em Curitiba ocorre sobre as espécies arbóreas nativas, sendo que $14,3 \%$ das espécies dos macroliquens ocorrem exclusivamente sobre estas árvores. Esta concentração de espécies é devida principalmente a Tabebuia chrysotricha e Syagrus romanzoffiana. Tabebuia chrysotricha apresenta sobre seu córtex $73,8 \%$ das espécies encontradas, enquanto Syagrus romanzoffiana apresenta $55,9 \%$. Estas forófitos apresentam grande similaridade florística $(0,73)$. Devido à capacidade de ambas as espécies vegetais portarem uma comunidade liquênica diversificada e à similaridade na composição desta comunidade, estes forófitos possuem grande potencial de utilização em estudos de mapeamento, podendo-se averiguar a correlação entre a diminuição de espécies de liquens a níveis crescentes de poluentes atmosféricos.

Apesar do potencial de Tabebuia chrysotricha e Syagrus romanzoffiana em estudos de mapeamento, de acordo com Milano et al. (1992), estas representam apenas $7,9 \%$ das árvores plantadas em vias públicas em Curitiba, o que limita sua utilização em projetos englobando toda a cidade. Por outro lado, Syagrus romanzoffiana é uma espécie comumente plantada em áreas centrais da cidade e também em vias de grande fluxo de tráfego, o que possibilitaria sua utilização pontual em regiões estratégicas da cidade.

Com relação às espécies arbóreas exóticas observase que apresentaram $66,7 \%$ da flora liquênica total, sendo que Ligustrum lucidum e Tipuana tipu concentraram $76,8 \%$ das espécies encontradas. Estas espécies possuem também grande similaridade florística com relação às espécies liquênicas $(0,79)$ e, por representarem $21,9 \%$ das espécies arbóreas plantadas em vias públicas em Curitiba (Milano et al., 1992), podem figurar entre as forófitos preferenciais em estudos de mapeamento nesta cidade.

Embora Lagerstroemia indica seja a espécie arbórea mais plantada em vias públicas em Curitiba (Milano et al., 1992), esta não deve ser incluída em estudos de biomonitoramento utilizando a comunidade liquênica, pois é a árvore com menor riqueza de espécies, portando apenas $26,2 \%$ das espécies. A maioria dos liquens encontrados sobre este forófito, o qual entre todos demandou o maior esforço amostral, foi coletada sobre ramos finos, pois esta árvore caracteristicamente perde o córtex, sofrendo um processo de descamação do tronco que impossibilita a permanência dos liquens.

Dentre as 84 espécies de liquens epífitas encontradas, 12 são capazes de crescer sobre todas as espécies arbóreas amostradas e somente três: Dirinaria confluens, Pyxine subcinerea e Ramalina celastri não foram observadas sobre uma espécie de forofófito (Tabela 1). Estes macroliquens, por não apresentarem preferência em relação à espécie arbórea, constituem-se em espécies recomendadas para a utilização em projetos de biomonitoramento em Curitiba, considerando que, como recomendado por Geebelen e Hoffmann (2001), a utilização de poucas espécies e não da flora liquênica total, propicia que pesquisadores não especialistas, ou seja, não liquenólogos, possam reconhecê-las em campo.

Além disto, entre estas 15 espécies, encontram-se liquens referidos na literatura (Hafellner et al., 1979; Estrabou, 1998) como nitrófilos, isto é, que crescem abundantemente em ambientes enriquecidos com compostos nitrogenados, a saber, Candelaria concolor e espécies da família Physciaceae: Dirinaria confluens, $D$. applanata, Heterodermia obscurata, Physcia poncinsii, e Pyxine subcinerea. Estas espécies poderiam ser utilizadas em projetos de biomonitoramento podendo ser avaliado se seu predomínio (abundância) em determinadas regiões da cidade estaria relacionado ao incremento da deposição de compostos nitrogenados, a exemplo de projetos desenvolvidos em outros países (Søchting, 1995; Carroll et al., 1999; Gombert et al., 2003).

Neste grupo de espécies não seletivas em relação aos forófitos encontra-se também Canoparmelia texana. Esta espécie é citada por Marcelli (1998) como tolerante a poluição, e vem sendo utilizada em estudos de poluição atmosférica em São Paulo (Saiki et al., 1997; 2001; Fuga et al., 2008). Canoparmelia texana também é apontada por Martins et al. (2008) como uma espécie característica de ambientes alterados em estudos de biomonitoramento utilizando liquens na região metropolitana de Porto Alegre. 


\section{Agradecimentos}

À Fundação Araucária pelo auxílio financeiro e bolsa de Iniciação Científica de Lucas Nogueira. Ao CNPq pela bolsa de Iniciação Científica de Patrícia Wolf Veiga. À Manuela Dal-Forno pela revisão do Abstract.

\section{Referências}

Brodo, I. M.; Sharnoff, S. D.; Sharnoff, S. 2001. Collecting and studying lichens. In: Brodo, I. M.; Sharnoff, S. D. \& Sharnoff, S. (Eds). Lichens of North America. Yale University Press, New Haven, USA, p. 97-109.

Carroll, J. A.; Caporn, S. J. M.; Cawley, L.; Read, D. J.; Lee, J. A. 1999.The effect of increased deposition of atmospheric nitrogen on Calluna vulgaris in upland Britain. New Phytologist, 141 (3): 423-431.

Culberson, C. F.; Ammann, K. 1979. Standardmethod zur Dünnschicht-chromatographie von Flechtensubstanzen. Herzogia, 5: 1-24.

Elix, J. A. 1994. Lichen chemistry and simple procedures for its application in the Parmeliaceae. In: Grgurinovic, C. (Ed.). Flora of Australia 55 Lichens - Lecanorales 2. Australian Biological Resources Study, Canberra, Australia, p.2-4.

Elix, J. A.; Ernst-Russell, J. L. 1993. A catalogue of standardized thin layer crhomatographic data and biosynthetic relationships for lichen substances. $2^{\text {nd }}$ ed. Australian National University, Canberra, Australia, 231pp.

Estrabou, C. 1998. Lichens species identification and distribution according tolerance to airborne contamination in the city of Córdoba, Argentina. In: Marcelli, M. P. \& Seaward, M. R. D. (Eds). Lichenology in Latin America: History, current knowledge and application. CETESB, São Paulo, Brasil, p.165-169.

Fuga, A; Saiki, M; Marcelli, M. P.; Saldiva, P. H. N. 2008. Atmospheric pollutants monitoring by analysis of epiphytic lichens. Environmental Pollution, 151 (2): 334-340.

Geebelen, W.; Hoffmann, M. 2001. Evaluation of bio-indication methods using epiphytes bycorrelating with $\mathrm{SO}_{2}-$ Pollution parameters. Lichenologist, 33 (3): 249-260.

Giordani, P. 2007. Is the diversity of epiphytic lichens a reliable indicator of air pollution? A case study from Italy. Environmental Pollution, 146 (2): 317-323.

Gombert, S.; Asta, J.; Seaward, M. R. D. 2003. Correlation between the nitrogen concentration of two epiphytic lichens and the traffic density in an urban area. Environmental Pollution, 123: 281-290.
Hafellner, J.; Mayrhofer, H.; Poelt, J. 1979. Die Gattungen der Flechtenfamilie Physciaceae. Herzogia, 5: 39-79.

Jeran, Z.; Mrak, T.; Jaćimović, R.; Batič, F.; Kastelec, D.; Mavsar, R.; Simončič. P. 2007. Epiphytic lichens as biomonitors of atmospheric pollution in Slovenian forests. Environmental Pollution, 146 (2): 324-331.

Marcelli, M. P. 1998. Hystory and current knowledge of Brazilian lichenology. In: Marcelli, M. P. \& Seaward, M. R. D. (Eds.). Lichenology in Latin America: History, current knowledge and application. CETESB, São Paulo, Brasil, p.25-45.

Martins, S. M. A.; Käffer, M. I.; Lemos, A. 2008. Liquens como bioindicadores da qualidade do ar numa área de termoelétrica, Rio Grande do Sul, Brasil. Hoehnea 35 (3): 425-433.

Milano, M. S.; Nunes, M. L.; Santos, L. A.; Sarnowski Filho, O.; Robayo, J. A. M. 1992. Aspectos quali-quantitativos da arborização de ruas de Curitiba. Anais do 1 Congresso Brasileiro sobre Arborização Urbana, 2: 199-211.

Munzi, S.; Ravera, S.; Caneva, G. 2007. Epiphytic lichens as indicators of environmental quality in Rome. Environmental Pollution, 146 (2): 350-358.

Pignata, M. L. 1998. Lichens and air pollution in Argentina. In: Marcelli, M. P. \& Seaward, M. R. D. (Eds.). Lichenology in Latin America: History, current knowledge and aplication. CETESB. São Paulo, Brasil, p.155-164

Poličnik, H.; Simončič, P.; Batič, F. 2008. Monitoring air quality with lichens: A comparison between mapping in forest sites and in open areas. Environmental Pollution, 151 (2): 395-400.

Saiki, M.; Chaparro C. G.; Vasconcellos M. B. A.; Marcelli M. P. 1997. Determination of trace elements in lichens by instrumental neutron activation analysis. Journal of Radioanalytical and Nuclear Chemistry, 217 (1): 111-115.

Saiki, M.; Horimoto L. K.; Vasconcellos M. B. A.; MarcellI M. P.; Coccaro D. M. B. 2001. Survey of elemental concentrations in lichen samples collected from Sao Paulo State. Journal of Radioanalytical and Nuclear Chemistry, 249 (2): 317-320.

Søchting, U. 1995. Lichens as monitors of nitrogen deposition. Cryptogamic Botany, 5 (3): 264-269.

Seaward, M. R. D. 2002. Lichens as monitors of air pollution at risby warren adjacent to scunthorpe steelworks, North Lincolnshire. Coke Oven Managers Association, 1: 127-144.

Sommerfeldt, M.: John, V. 2000. Evaluation of a method for the reassessment of air quality by lichen mapping in the city of Izmir, Turkey. Turkey Journal of Botany, 25 (2001): 45-55. 